\title{
Anisotropic Diffusion based Brain MRI Segmentation and 3D Reconstruction
}

\author{
M. Arfan Jaffar ${ }^{1}$, Sultan Zia ${ }^{1}$, Ghaznafar Latif ${ }^{1}$ \\ ${ }^{I}$ National University of Computer \& Emerging Sciences, Islamabad \\ Pakistan \\ arfan.jaffar@nu.edu.pk, ziactn@.gmail.com, ghazanfar.latif@gmail.com, \\ Anwar M. Mirza ${ }^{2}$ \\ ${ }^{2}$ College of Science Research Centre, King Saud University, Riyadh \\ Saudi Arabia \\ ammirza@ksu.edu.sa, \\ Irfan Mehmood ${ }^{3}$, Naveed $\mathrm{Ejaz}^{3}$, Sung Wook Baik ${ }^{3 *}$ \\ ${ }^{3}$ College of Electronics and Information Engineering, Sejong University, Seoul \\ South Korea \\ irfanmehmood@sju.ac.kr, naveed@sju.ac.kr, sbaik@sejong.ac.kr \\ Received 6 April 2011 \\ Accepted 23 March 2012
}

\begin{abstract}
In medical field visualization of the organs is very imperative for accurate diagnosis and treatment of any disease. Brain tumor diagnosis and surgery also required impressive 3D visualization of the brain to the radiologist. Detection and 3D reconstruction of brain tumors from MRI is a computationally time consuming and error-prone task. Proposed system detects and presents a 3D visualization model of the brain and tumor inside which greatly helps the radiologist to effectively diagnose and analyze the brain tumor. We proposed a multi-phase segmentation and visualization technique which overcomes the many problems of $3 \mathrm{D}$ volume segmentation methods like lake of fine details. In this system segmentation is done in three different phases which reduces the error chances. The system finds contours for skull, brain and tumor. These contours are stacked over and two novel methods are used to find the 3D visualization models. The results of these techniques, particularly of interpolation based, are impressive. Proposed system is tested against publically available data set [41] and MRI datasets available from MRI \& CT center Rawalpindi, Pakistan [42].
\end{abstract}

Keywords- Magnetic resonance imaging (MRI), Classification, Image Segmentation,Tumor detection , 3D reconstruction

\section{Introduction}

In this paper, we propose a fully automatic and efficient algorithm for realistic $3 \mathrm{D}$ brain tumor model reconstruction by using multiple 2D MR images. Segmentation and 3D volume reconstruction in medical imaging is a tool allowing a diagnostics automation and as well will assist the expert in the qualitative and quantitative analysis. It's an important step in various applications such as visualization, quantitative analysis and image-guided surgery. 3D segmentation of pathology and healthy structures is extremely important for surgical planning, qualitative and quantitative analysis such as volume measurements. Precise segmentation of pathological structures is a difficult task because brain tumors vary greatly in size and position may have overlapping intensities with normal tissue and may be space occupying.

*Corresponding Author: Sung Wook Baik, Sejong University, Seoul, South Korea, sbaik@sejong.ac.kr

Published by Atlantis Press

Copyright: the authors 
Automatic segmentation methods of MRIs mostly leave segmentation errors. These errors of segmentation are removed interactively using computer graphics tools. This is performed by operating on individual 2D slices. Even advanced 2D tools required this daunting task [24]. 3D versions of segmentation corrections tools like [25] also limited the user interaction to $2 \mathrm{D}$ volume sections. But 3D view is necessary to see some artifacts which are not possible by individual 2D slice. 3D Volumetric segmentation methods give although a good overall picture of the data set, but they often appear to be confusing and lacking fine details. Moreover, for surface rendering the brain surface is usually not directly available in the original 3D volume MRI data [24]. Therefore, 3D visualization techniques still need a significant research and development challenge. We proposed a multi-phase segmentation and visualization technique which is suitable for above mentioned problems and covers the deficiencies of 3D volumetric segmentation methods.

Computer aided surgeries are playing an effective role in the medical field. Brain surgery is one of the very sensitive surgeries as brain has very delicate and complex structure. Another obstacle in the brain study is the protective skull part. Brain normal structure can be altered due to a disease known as brain tumor. A tumor is a mass of tissue that grows out of control of the normal forces that regulates growth [1]. Brain tumor causes the abnormal growth of the cells in the brain. The cells which supplies the brain in the arteries are tightly bound together there by routine laboratory test are inadequate to analyze the chemistry of brain [2]. Imaging modality Magnetic Resonance Imaging (MRI) provides high contrast images of the internal structure of the brain as compared to any other medical imaging modality. Brain tumor diagnosis is possible by analyzing these brain MR images. But this is a very critical task to accurately identify the tumor cells inside the affected brain. Segmentation is performed on these images for tumor region extraction. Image segmentation is a process of partitioning an image into different homogeneous regions, so that meaningful information about the image can be obtained and different analysis can be performed on that segmented image. We applied segmentation on MR images in three different phases. By this we achieve high accuracy in segmentation and tumor detection. We start tumor segmentation only when we left with brain part of the MR images. Extraction of brain tumor region requires the segmentation of brain MR images into two segments. One segment contains the normal brain cells and the second segment contains the tumorous cells of the brain. Correct segmentation of MR images is very important because most of the time these segments can be easily overlapped with each other.

\section{Major Contributions:}

In this paper, we proposed a system that provides $3 \mathrm{D}$ visualization system of the brain and of tumor inside it. Proposed system uses brain MR images for this application. This system is composed of multiple phases. First phase of the proposed system removes background from MR image and finds the head mask and skull boundary. The head mask is given as input to the second phase. In this phase first we construct an initial brain mask which is finalized by an active contour algorithm. So we extract the final brain mask and brain boundary. The tumor region is extracted from this brain part of the images. We found that level set evolution combining global smoothness with the flexibility of topology changes offers significant advantages over the conventional statistical classification followed by mathematical morphology. We used a modified fully automated competition based level set snake method to segment the tumor from brain cells. The tumor region which is extracted from this phase is used for 3D visualization. We used rendering algorithm for 3D visualization of brain and tumor.

The paper is organized as follows. Section II contains related work carried out in this field and identifies the problems associated with this field. Detail description of the proposed system is described in Section III. Section IV contains the details of implementation and relevant results. Finally, conclusions and discussions are presented in Section V.

\section{Related work}

The problem of automating 3D segmentation of brain imaging using Magnetic Resonance Imaging or other modalities, has received special consideration and hence there is numerous published research work $[3,4]$ in this field. One of the reasons is the multi benefits that may be gained from automated 3D brain segmentation. In literature a good deal of research work is available for 3D brain MRI tissue segmentation. They are broadly classified into three frameworks: contour-based, region-based and classification based segmentation [5]. In contour-based segmentation the active contour algorithm is a typical and widely used in literature [6] [7]. Secondly in region-based segmentation many techniques have been developed like region growing technique [8], split-and-merge based technique [9], and watershed based technique [10]. And Finally Classification-based segmentation includes statistical based classification such as in [11] Markov random field is used, in [12] Hidden Markov models based technique is proposed, and in [13] Gaussian mixture model segmentation is proposed. The other sub categories of classification based segmentation are supervised learning based segmentation and unsupervised learning based segmentation. Neural networks [14], support vector machine [15] are categorized as 
supervised where as fuzzy Cmeans (FCM) [16], spectral clustering [17], spatial fuzzy Cmeans[18] are the examples of unsupervised segmentation. It has been seen that in general, supervised segmentation methods could get good performance while unsupervised learning without any prior knowledge will get limited performance [19]. On the other hand supervised techniques require experts to supply labeled pixels to the model, which is expensive and time-consuming and unsupervised techniques do not use prior knowledge even if it's available. So we proposed a multi-phase technique to take the maximum of benefits while keeping it fully automatic. BET (Brain Extraction Tool) [20] is a popular technique used to extract the brain from non-brain tisues. It is used in SIENA (Structural Image Evaluation, using Normalisation, of Atrophy), which is considered a robust approach to quantifying brain volume change [21]. An extension of BET to remove segmentation imperfections (e.g. incorrect inclusion of eyes, peri-orbital fat and other non-brain structures) is proposed in [22]. However, the methods [20,22] cannot always be used directly for removing non-brain tissues in brain MRI[23]. For classification of brain images as normal and abnormal El-Syed et al [26] proposed a hybrid technique. In this technique first features of the brain MR image are extracted using Discrete Wavelet Transform (DWT) which are then reduced using PCA. Last step is classification based on these features. For MRI brain image segmentation Sripama [27] proposed fuzzy symmetry based genetic clustering technique. In [27] numbers of clusters are evolved by variable length genetic fuzzy clustering technique. For measuring quality of cluster fuzzy point symmetry based cluster validity index is proposed in this paper. Experiments are performed on different T1, T2 and PD brain images. This technique performs better then FCM and Expectation Maximization algorithm. But this technique does not consider spatial information and sometime does not segment brain image correctly. This technique also does not work properly for the data sets which have same point as a center for different clusters. Fuzzy C-means clustering is a clustering technique which clusters the similar objects into similar regions. FCM with spatial information has been successfully applied for color image segmentation in [18].

Zhang proposed a Hidden Markov Random Field Model and the Expectation-Maximization algorithm for segmentation of brain MR images [28]. This is a fully automatic technique for brain MR images segmentation. This method is based on estimation of threshold that is heuristic in nature. Thus most of the time, this method does not produce accurate results. Method in [28] is also computationally very expensive. Rajeev et al [29] proposed a method for brain tumor detection based on segmentation of the image. Segmentation of the brain MRI is done using watershed algorithm in the proposed technique.
The method in [29] does not require any initialization for the segmentation of the brain MRI. Dubey et.al [30] proposed a semi-automatic segmentation technique for brain tumors from MRI. Level set evolution with contrast propagation is used for segmentation in [30]. Pre and post contrast difference images and mixture modeling fit of the histogram are used for calculating probabilities for background and tumor region. For segmentation of the tumor boundaries level set evolution is initialized using whole image.

\section{PROPOSED SYSTEM}

Taking MRI scans produce pictorial representation of processes, forming a complete image of a brain. In order to perform detailed studies of these images we need to distinguish the patterns of brain and segmentation of brain from the rest of the head from its MRI.

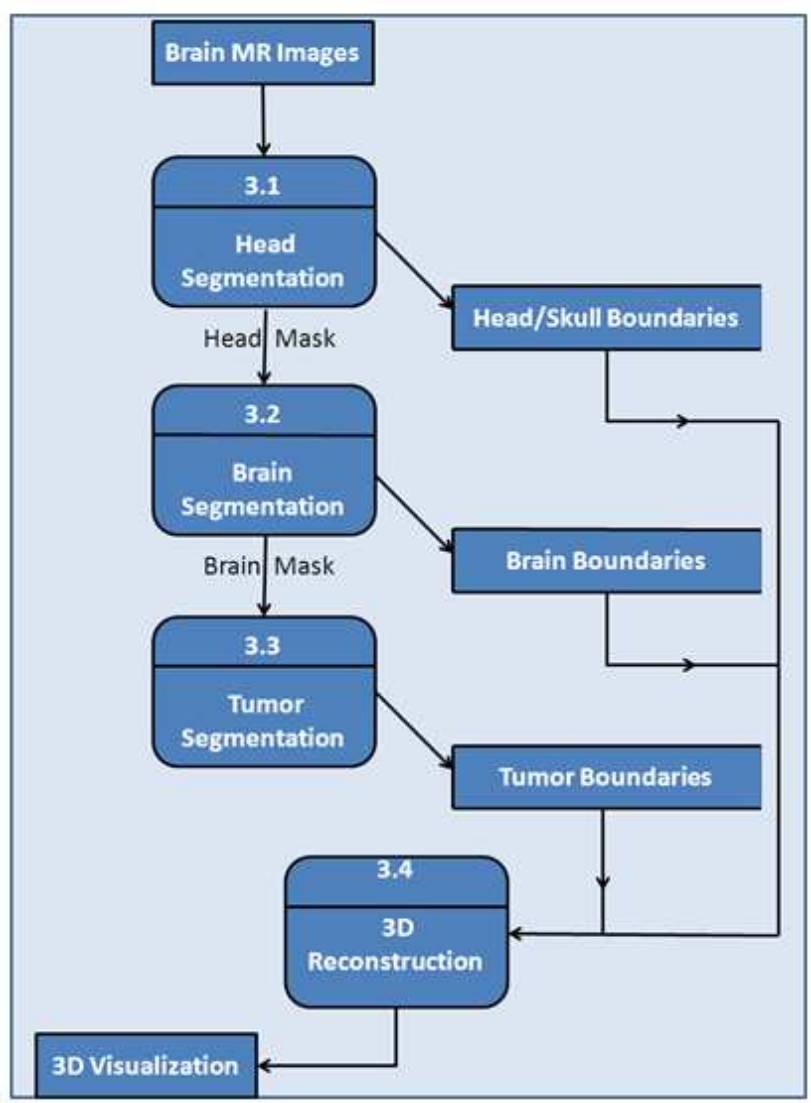

Fig.1 Architecture of the Proposed System

Here is the methodology adopted for distinguishing the brain from the rest of the image. The proposed system consists of four major modules. Brain MRIs are provided to the system as an input. First module consists of head segmentation and finds the skull/head boundary from input MR image. Second module of the proposed system creates a brain mask then finds 
brain boundary and separates the brain part of MR image. Third module of the proposed system is segmentation of the brain as extracted in the previous module. As a result we extract the tumor and find its boundary. Segmentation extracts the tumor region from the brain MR image. From the information of these segmentations 3D model of the skull, brain and brain tumor is constructed. Top level system architecture of the proposed method is shown in Fig.1. Details about the major components of the proposed algorithm are discussed in the following subsections one by one.

\section{A. Head Segmentation}

Owing to the high similarity between the gray levels of the brain MR image and the image background (see Fig.2 (a)), we cannot eliminate background by simply applying threshold to the image. For this reason we used an operator, starting from the corners of the image and moves along the two opposite directions identifying as background pixels. These background pixels with a gray level within a range fixed in the configuration phase of the system [31] can be separated from the rest of the image. Now we can get an initial level head mask by converting it to a binary image. Threshold would produce speckles inside and outside the mask so we use morphological operation (erosion and dilation) to get a perfect binary image without any noise and distortions. This is shown in Fig.2 (b). In third step we generate the boundary of the head mask. For that purpose we use the image under application of erosion and subtract it from the mask to get a boundary of the head. The detected head boundary is shown in Fig.3 (b).
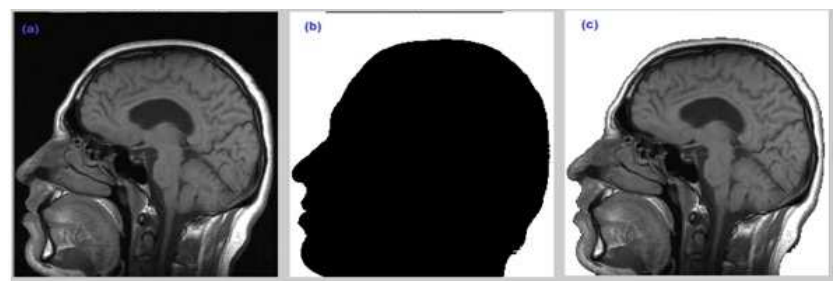

Fig.2 (a) Original Image, (b) Binary Head Mask, (c) Segmented Head Image

\section{B. Brain Segmentation}

After the head segmentation we need to generate an initial brain mask that would help us to distinguish only the brain part from the rest of the head's image. It uses anisotropic diffusion and then Gaussian filter will be applied to the background noise removed image of the head. Then centering and locating origins on the image will help to detect only the brain part. First of all we applied the anisotropic diffusion.

This is a type of filter applied on images to make their edges smoother and fuzzier. A diffusion function is used to monotonically degrade the image gradient. The process consists of updating each pixel of the image by an amount equal to the flow contributed by the four nearest neighbors of the pixels. We used 2-D anisotropic diffusion to attenuate the non-brain part. This enables the brain to be segmented using a simple threshold.
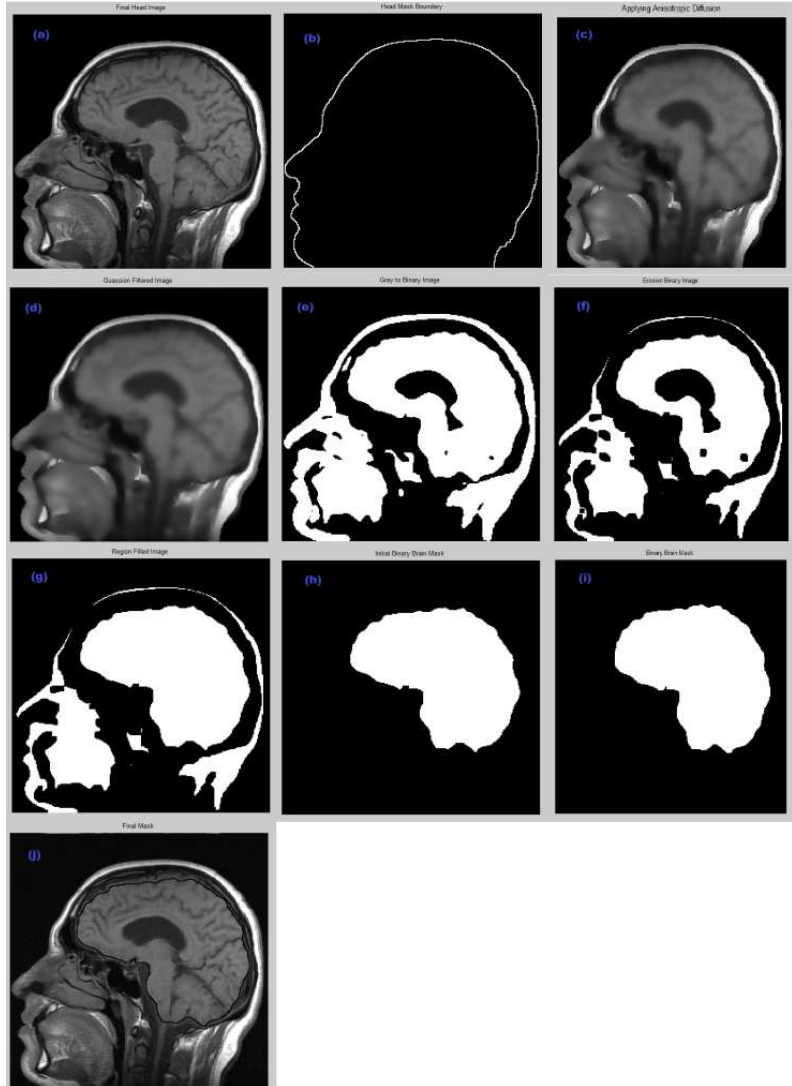

Fig.3 (a) Noised Removed Image, (b) Head Mask Boundary, (c) Applied Anisotropic Diffusion, (d) Applied Gaussian Filter, (e) Binary Conversion, (f) Applied Erosion, (g) Region Filled, (h) Eliminating non brain parts, (i) Applied dilation, (j) Initial Brain Mask

Perona and Malik [32] formulated the anisotropic diffusion filter as a diffusion process that encourages integration smoothing while inhabiting integration smoothing:

$$
\frac{\partial}{\partial t} I(\bar{x}, t)=\nabla \cdot(c(\bar{x}, t) \nabla I(\bar{x}, t))
$$

In our case $I(\bar{x}, t)$ is the MR Image. $\bar{x}$ refers to the image axes (i.e $\mathrm{x}, \mathrm{y})$ and $t$ refers to the number of iteration steps. $c(\bar{x}, t)$ is called the diffusion function. We use the following diffusion function:

$$
c(\bar{x}, t)=\exp \left(-\left(\frac{\nabla I(\bar{x}, t)}{\sqrt{2} k}\right)^{2}\right)
$$

$K$ refers to as the diffusion constant and the behavior of filter depends upon the value of K. Fig.3 (c) shows the resultant image after applying the anisotropic diffusion. 
We segment the brain using a threshold value which is found by fitting a Gaussian curve to the histogram of the diffused volume. The Fig.3 (d) shows the resultant image after applying Gaussian filter [33]. After applying the Gaussian filter, we convert the gray level image into binary level image by using automatic threshold technique. It is shown in the Fig.3 (e). Erosion is performed on the image before filling the gaps and spaces. This erosion cuts the portion of the image of eyes and other non-brain parts and what we get is a distinguished brain shape. Fig.3 (f) shows the resultant image after applying erosion.

The binary mask produced by automatic threshold has gaps; these gaps are filled by using morphological operations. The resultant image is shown in Fig.3 (g). Once these parts are separated from the brain they are made to disappear using spatial information. Here we define origin in the brain part of the image. Using that origin as reference point we eliminate those areas that do not fall under the brain part and are irrelevant for our use. The resultant image is shown in Fig.3 (h). Having separated the brain image from the original we need to dilate it in order to get a bigger image.

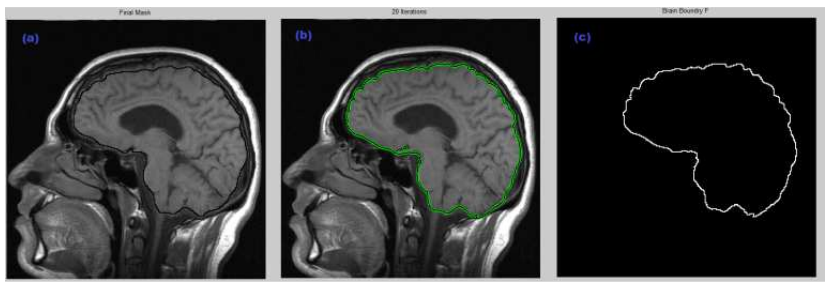

Fig.4 (a) Initial Brain Mask, (b) Final Brain Mask, (c) Brain Boundary

This image would be subtracted from the eroded mask we just found. This is how we get the boundary of the brain finally. The resultant image is shown in Fig.3 (i). Now we can map the boundary on the original image. We can also extract the boundary of the initial brain mask. The Fig.3 (j) shows the initial brain mask. But this still has a problem. The mask that is produced doesn't fit very nicely on the brain part. There are some parts of the brain that fall outside the boundary. To eradicate this problem another process is applied. We use active contour model algorithm introduced by Kass et al [34] and the results are shown in fig.4.

\section{Tumor Segmentation}

Tumor segmentation is performed for extracting the tumorous brain portion from the brain MRI. Segmentation process segments the brain MR image into two portions. One segment contains the normal brain tissues and the second segment contains the tumorous cells. The segment which contains the tumorous cells is the desired region which is known as timorous region. Dubey et el [30] has proposed a tumor segmentation method for $3 \mathrm{D}$ MR images. It performs well to find the tumor but appears to be confusing and lacking fine details and becomes inefficient due to the $3 \mathrm{D}$ operations and initialization with the whole MR image.

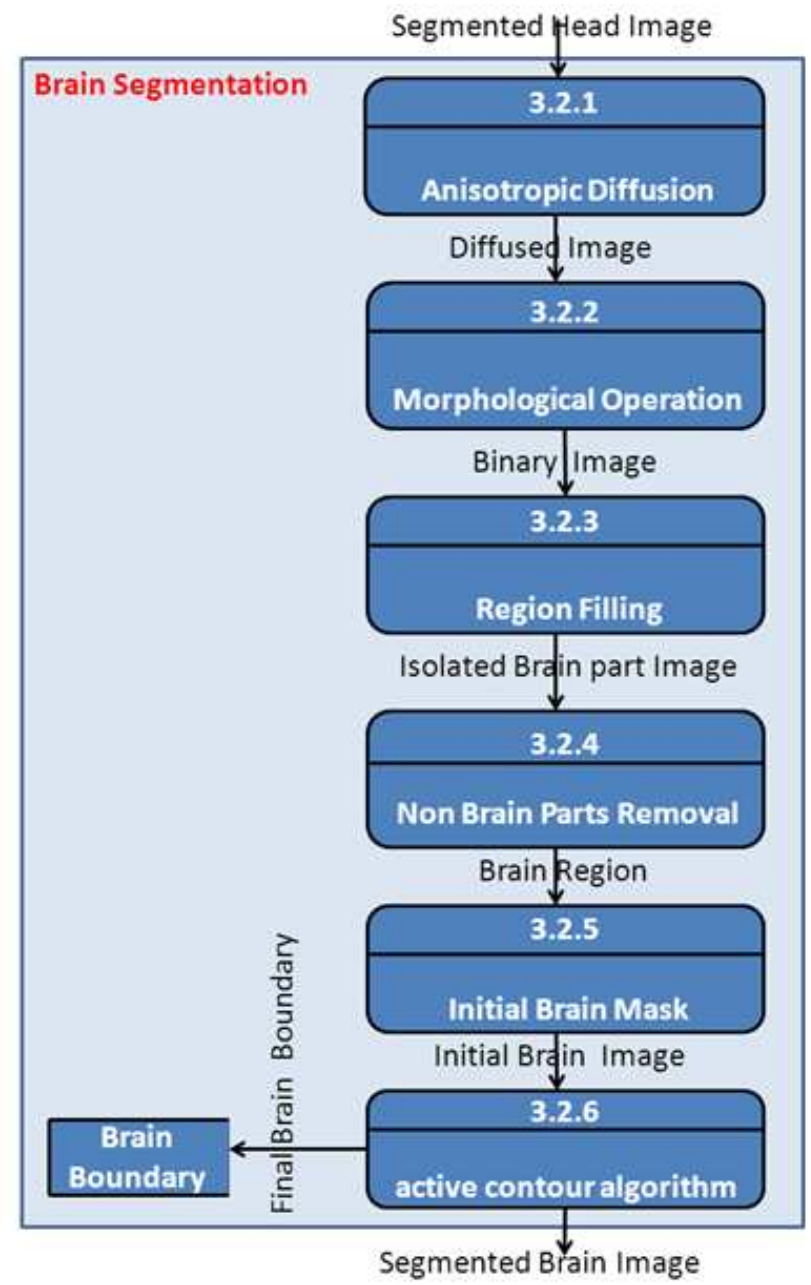

Fig.5 Architecture of the Brain Segmentation Module

We applied this method in 2D environment and used only brain part of the image for initialization so get quick convergence and achieved efficiency. Moreover, since we applied the tumor segmentation only on the brain part of the image, the error chances of [30] reduced to large extant. The method starts with an intensity-based fuzzy classification of pixels into tumorous brain and normal brain classes. The tumorous brain probability map is used to locally guide the propagation direction and speed of a level-set snake. To initialize the snake the tumorous probability map is used which derives it automatic. Image forces are balanced with global smoothness constraints to converge stably to a smooth tumor segmentation of arbitrary topology. The probability 
map gives the probability that the given pixel belongs to the tumor or not is a scalar field evaluated pixel by pixel. The histogram of the difference image (pixel-by-pixel) is defined which clearly shows a symmetric distribution around zero and a second distribution is defined related to regional changes caused by the contrast agent. We defined the histogram by a mixture density of two distributions, a Gaussian function to model small differences around zero and a Poisson distribution to model the changes due to contrast. Then we used the nonlinear fit package provided by mathematics. The scalar field derived from the posterior probability with range [$1,1]$ is passed to the level-set algorithm as the probability map $\mathrm{p}$ (A) - p (B). The level-set snake uses the fuzzy classification for its image forces with addition smoothness constraints [35].

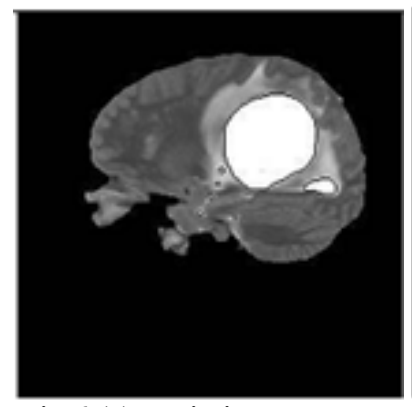

Fig.6 (a) Brain image

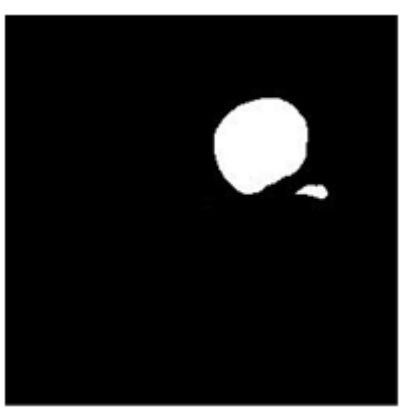

(b) Segmented Tumor image
In order to make Initialization of snake completely automatic and without the intervention of the user, we used competition snake which is more robust to variable initialization. A level zero set of the tumor probability map, where $\mathrm{P}$ (tumor) $=\mathrm{P}$ (non-tumor), is used as the initialization. The implicit function is initialized to the distance map of the initial contour.

Because of the stability the level set method has been used to capture rather than track interfaces. The level set snake is defined as the zero level set of an implicit function $\varphi$ defined on the entire image. The evolution of the level set snake is considered via a partial differential equation of the implicit function $\varphi$ and propagates to its boundary uniformly at a constant speed $\psi$ :

$$
\frac{\partial \varphi}{\partial t}=\psi|\nabla \varphi|
$$

The deformation model is driven by image forces and constrained by prior information on the shape of the model. In the partial differential equation governing the evolution of the implicit function of snake the image forces and smoothness constraints are simply separate terms. The region competitionbased snake uses image forces to change the direction of propagation. The snake shrinks when the boundary encloses parts of the background (B) and grows when the boundary is inside the tumor region (A):

$$
\frac{\partial \varphi}{\partial t}=\psi[\mathrm{p}(\mathrm{A})-\mathrm{p}(\mathrm{B})]|\nabla \varphi|
$$

If the snake is guided by image forces there are chances of leak into many small noisy structures that are not part of the tumor. So to balance the forces we used the mean curvature flow to the snake contour. In the level-set formalism this is easily done by adding a term $\left(\mathrm{C}_{\mathrm{mcf}}\right)$ to the snake evolution equation.

$$
\begin{aligned}
\frac{\partial \varphi}{\partial t} & =\psi[\mathrm{p}(\mathrm{A})-\mathrm{p}(\mathrm{B})]|\nabla \varphi| \\
& +C_{m c f} \nabla \cdot\left[\frac{\nabla \varphi}{|\nabla \varphi|}\right]|\nabla \varphi|
\end{aligned}
$$

Finally, a smoothing term is added to the implicit function in order to gain numerical stability of the algorithm. So, to ensure numerical stability of the forward-in-time, centered-inspace solution of the partial differential equation, we apply an additional constant factor $\mathrm{C}_{\mathrm{sf}}$ to control the strength of this smoothing:

$$
\begin{array}{r}
\frac{\partial \varphi}{\partial t}=\psi[\mathrm{p}(\mathrm{A})-\mathrm{p}(\mathrm{B})]|\nabla \varphi| \\
+C_{m c f} \nabla \cdot\left[\frac{\nabla \varphi}{|\nabla \varphi|}\right]|\nabla \varphi| \\
+C_{s f} \nabla^{2} \varphi
\end{array}
$$

When using the level set method in image segmentation, an initial front should be chosen appropriately and let to propagate it with a speed function. Dubey et el [30] provide a semi automatic method and required a user intervention to define a front. We provide a fully automated method with no user intervention by defining an appropriate front by using SFCM [18]. The level set splits the space into inside and outside regions. The front propagates itself inside the image adapting itself to the boundary of the tumor. This technique solves two of the problems of snakes [24]: firstly, it allows the segmentation of objects with many bifurcations and protuberances and recovers complex shapes inside the image. Secondly, it is not necessary to know a priori the topology of an object in order for it to be recovered. Thirdly, there is no need of user intervention. The results of this method are shown in fig. 6.

\section{3D Reconstruction}

In computer graphics, 3D modeling is the phenomenon of developing a mathematical representation of any 3D objects through specialized software and programming languages. It can be displayed as a 2D image through a process called 3D rendering or by computer simulation of physical phenomena. Triangular meshing is used for $3 \mathrm{D}$ reconstruction from $2 \mathrm{D}$ 
contours using a sequence of $2 \mathrm{D}$ contours, detected by $2 \mathrm{D}$ segmentation method in the parallel cross-sectional MRI images. Due to complexity and irregular nature of each encephalic tissue boundary, three-dimensional (3D) reconstruction for MRI image has been a hot area. The given 3D model can be used to diagnose/visualize the brain tumor through the segmentation of MRI scan. We have to use MRI scan of up to 100 to 250 images from different dimensions of brain. The greater the numbers of MRI scan images the greater will be precision of the model and it will look more realistic. We generate skull, brain and tumor's boundary data files for $3 \mathrm{D}$ visualization. These boundary data files are used to make 3D model. Interpolation technique based on triangular meshing is used in creating models of skull, brain and tumor.

We propose a novel technique to construct a $3 \mathrm{D}$ model from $2 \mathrm{D}$ images. It is a robust and efficient technique which gives accurate results. The input to this module of our proposed system is the segmented MR images. We use 100 to 250 images from different dimensions of head like axial Images, saggital Images, coronal Images. More the number of images of MRI scan more precise the model will be. We constructed 3D models for skull, brain and tumor and can be seen immediately by making skull and brain voxels transparent. The first step to construct the 3D models is the detection of skull boundaries, brain boundaries and tumor boundaries. We used threshold to detect boundaries and then applied eroding to get one pixel wide boundary as it gives us the exact direction and number of pixels on boundary. It also eliminates abnormalities in modeling. If we do not read pixels in appropriate order then meshing cannot be performed accurately [32]. The skull boundaries, brain boundaries, and tumor boundaries are separated. These boundaries are represented by $\mathrm{SBi}, \mathrm{BBj}$ and $\mathrm{TBk}$ respectively. Here $\mathrm{i}, \mathrm{j}$ and $\mathrm{k}$ are the indexes over total number of skull boundaries, brain boundaries and tumor boundaries respectively. These three kinds of images are shown in fig. 11.
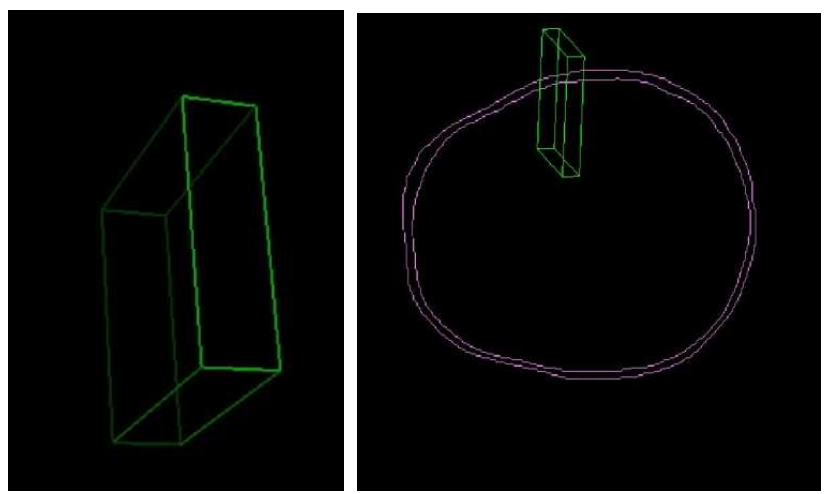

Fig.7 Volume Element
In meshing we have used triangles because they give more smoothness in modeling as compared to other geometric figures [36]. We have used two methods to connect neighboring points more precisely .We will explain them in more detail in later discussion. A volume containing voxels can be visualized either by direct volume rendering or by the extraction of polygon iso-surfaces which follow the contours of given threshold values. This uses dataset of multiple images in the form of 2D; combine these images in a sequence that represents 3D model [37]. In this method we have used a volume element as shown in fig.7. This volume element has dimension as $\mathrm{x}$ belongs to $[\mathrm{x}, \mathrm{x}+\mathrm{x}$ increment $]$ and $\mathrm{y}$ belongs to [image height/2,0]. Width of this volume element can be adjusted to find best matching neighbors. Algorithm 1 is developed using this technique (fig.8).

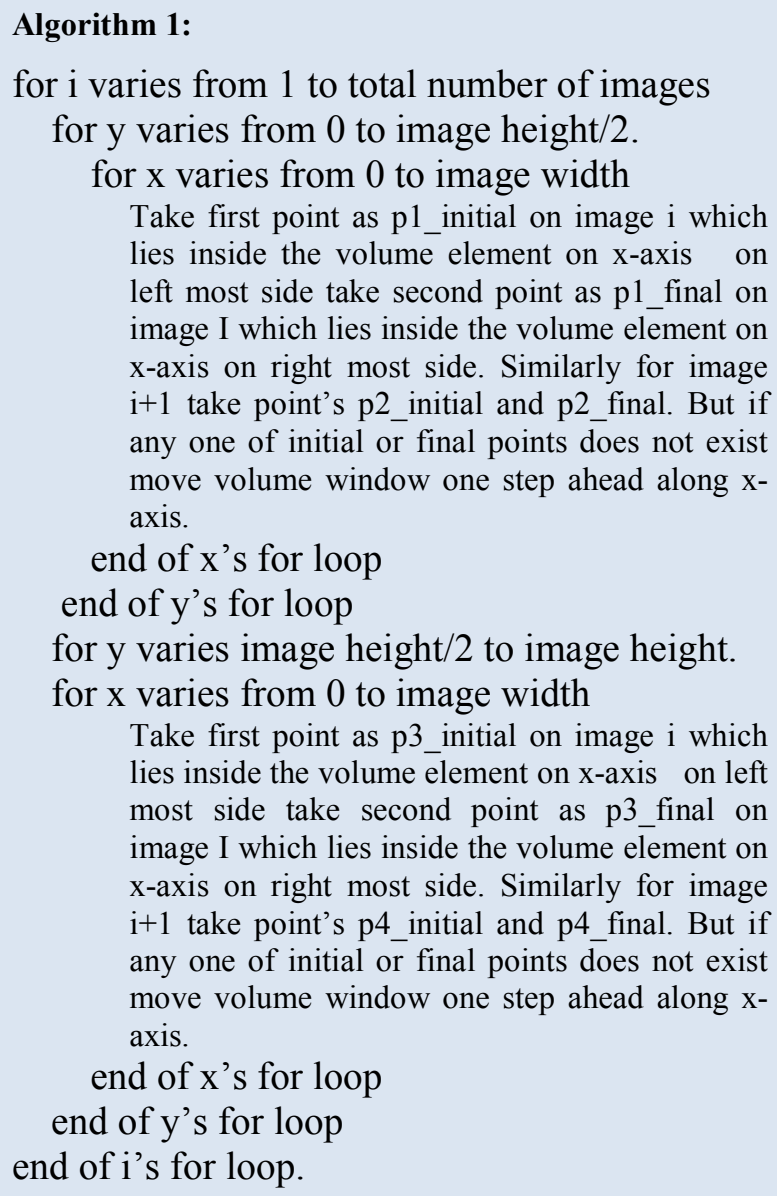
lies inside the volume element on $\mathrm{x}$-axis on left most side take second point as p3 final on image I which lies inside the volume element on $\mathrm{x}$-axis on right most side. Similarly for image i+1 take point's p4_initial and p4_final. But if any one of initial or final points does not exist move volume window one step ahead along $\mathrm{x}$ axis.

end of $x$ 's for loop

end of y's for loop

end of i's for loop.

Fig. 8 Algorithm to combine contour points using Volume Element to build 3D mesh

This gives good results and chooses neighboring points more accurately and efficiently but it has problem in certain situation where it has to deal with pixels lying on vertical lines. Because it is picking points in an interval of length 
$\mathrm{x}$ increment $=2$ or 3 pixels. The result of this method is shown in fig.9 (a).

It is not a good choice to simply connect the all points on boundary of one image to all points of boundary of image lying below or up of this image. Because this will give really bad result and model will not look realistic. Now the points on each contour are stored in text files so that these contour points are easily available while meshing. The skull, brain and tumor contour sizes increase or decrease in certain ways. For example if we are reading images from top to bottom of head then points on contours of images increases and at a certain level number of points on boundaries start decreasing.
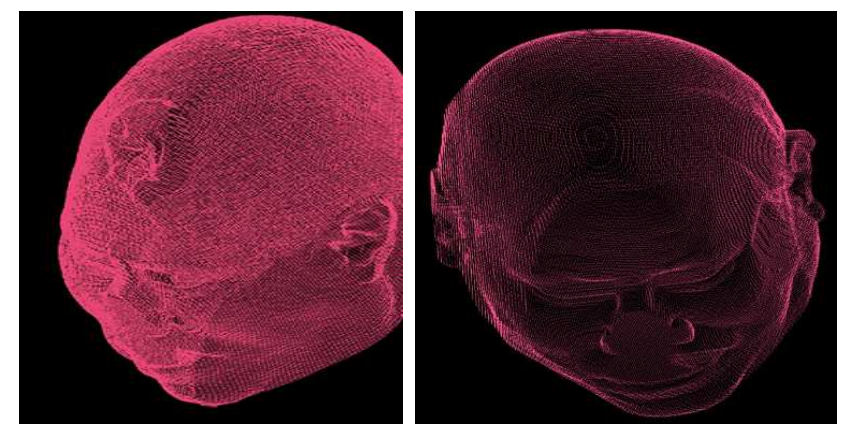

Fig.9 (a) Volume Element Working (b) Interpolation Working

While points on the contours of tumor can increase or decrease abruptly depending on the shape of tumor. Thus we need to find a way to efficiently connect these different numbers of points.

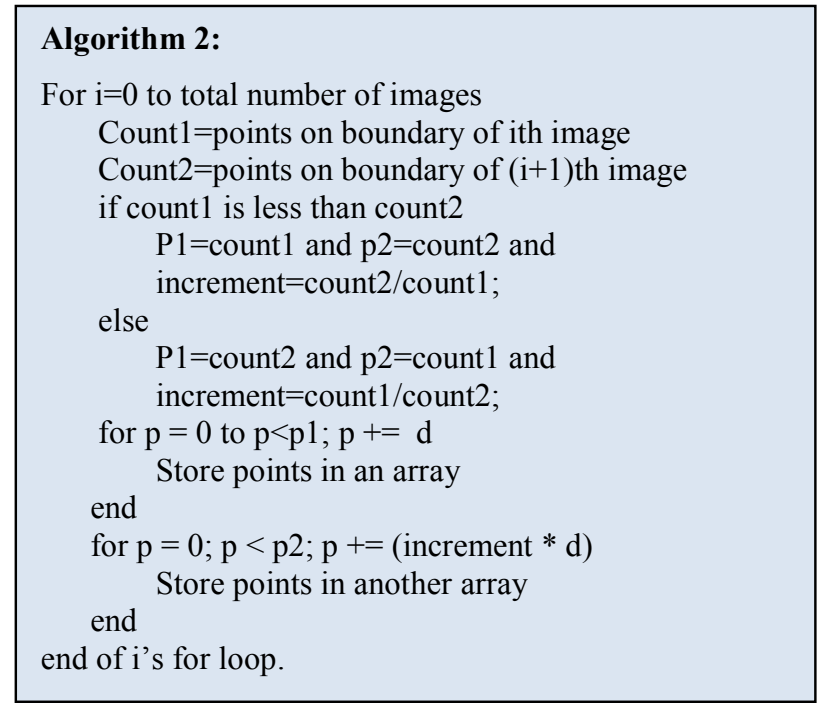

Fig.10 Algorithm to combine contour points using interpolation to build 3D mesh

The next points to connect on two consecutive contours can be calculated as:

$$
\begin{aligned}
& T B_{i, j+1}=T B_{i, j}+d \\
& T B_{i+1, j+1}=T B_{i+1, j}+\nabla d^{*} d \\
& \nabla d=\frac{n T B_{i+1}}{n T B_{i}}
\end{aligned}
$$

Where $d$ is a user defined incremental value and $n T B_{i}$ is number of points on ith tumor boundary.

Since $\nabla d$ is a decimal number, $T B_{i+1, j+1}$ is not necessarily an integer so we can use the flour of $T B_{i+1, j+1}$. Thus to overcome the above mentioned problem we have used interpolation to map points on boundary of one image to points on boundary of another image [39]. This approach is presented in algorithm 2 (fig.10).

Now we have equal number of points for both corresponding images. Connect them to form faces and find their normal. This gives good results as it maps all points on boundaries accurately. The result of this method is shown in fig.9 (b). Vertex normals are calculated instead of face normal as they facilitate later in clipping and shading [38]. Normal vector for a vertex ' $v$ ' of a face ' $f$ ' is $M^{f, v}$ can be calculated by Martin Newell's method:

$M^{f, v}=\left(M_{x}^{f, v}, M_{y}^{f, v}, M_{z}^{f, v}\right)$

Where

$$
\begin{aligned}
& M_{x}^{f, v}=\sum_{i=0}^{N-1}\left(y_{i}-y_{\text {next }(i)}\right)\left(z_{i}+z_{\text {next }(i)}\right) \\
& M_{y}^{f, v}=\sum_{i=0}^{N-1}\left(z_{i}-z_{\text {next }(i)}\right)\left(x_{i}+x_{\text {next }(i)}\right) \\
& M_{z}^{f, v}=\sum_{i=0}^{N-1}\left(x_{i}-x_{\text {next }(i)}\right)\left(y_{i}+y_{\text {next }(i)}\right) \\
& n \operatorname{ext}(i)=(i+1) \bmod N .
\end{aligned}
$$

Where $N$ is the number of vertices in the face $f$, in our case $N$ $=3$ and $\left(x_{i}, y_{i}, z_{i}\right)$ is the position of ith vertex. In the case of 3D graphics, rendering may be done slowly, as in prerendering, or in real time. Pre-rendering is a computationally intensive process that is typically used for movie creation, while real-time rendering is often done for $3 \mathrm{D}$ video games which rely on the use of graphics cards with $3 \mathrm{D}$ hardware accelerators. We have used real time rendering [40]. After generating 3D model of head we apply rendering technique on that model so it becomes more visible and real. We use lighting and shading in our models. This finally helps the experts to diagnose and find any tumor by providing more clear images of brain parts. The final results are shown in fig. 12 .

\section{Experimental Results and Discussion}

The proposed system is implemented by using the MATLAB 7.9.0.529(R2009b) and Dot net 2008 environment. 
Proposed technique is tested on the dataset available at [41] and it is also tested on real brain MRI data set available at [42]. Fig. 11 shows the complete procedure of development of 3D model of the skull, brain and tumor inside the brain. The complete results of each phase of the system and discussions are presented in every phase. The results of head segmentation and boundary detection are presented in first phase, in the second phase the results of brain segmentation and brain boundary detection are discussed in phase three. In third phase we present the tumor boundary detection and finally the $3 \mathrm{D}$ reconstruction in fourth phase.
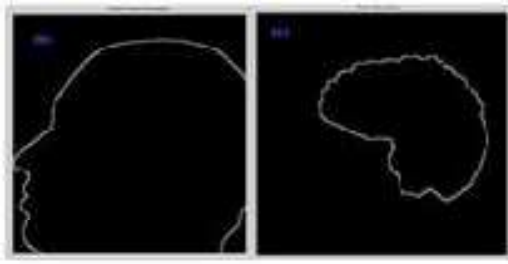

Input skull boundaries

Input brain

boundaries

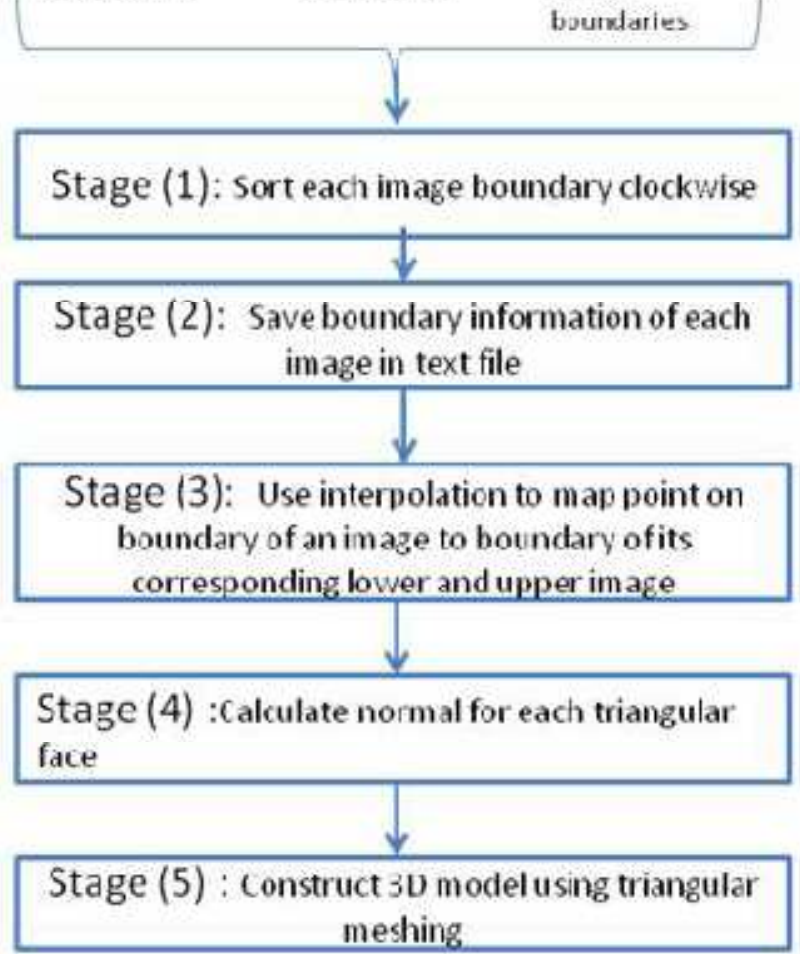

Fig.11 Process of development of 3D models

In fig. 11 we show how we developed the $3 \mathrm{D}$ models from these three kinds of contours. From all scans of a record of a patient brain mask, tumor mask, brain mask boundaries and tumor mask boundaries are obtained which are further used for 3D visualization. At stage 3 (fig.11), a volume based technique is also used along with interpolation. From these mesh models rendered 3D models are obtained by using real time rendering. The complete results for all phases are presented in fig. 12 below which are self explanatory.

\section{Conclusion and Future Work}

We developed an automated system which generates 3D model of brain and brain tumor from brain MR images. This proposed system is a multi-phase system that diagnoses the brain tumor and develops a $3 \mathrm{D}$ visualization model for brain. We applied segmentation on MR images in three different phases. By this we achieve high accuracy in segmentation and tumor detection. We start tumor segmentation only when we left with brain part of the MR images. In this paper, we proposed a system that provides $3 \mathrm{D}$ visualization system of the brain and of tumor inside it. This system is composed of multiple phases. First phase of the proposed system removes background from MR image and finds the head mask and skull boundary. The head mask is given as input to the second phase. In this phase first we construct an initial brain mask which is finalized by an active contour algorithm. So we extract the final brain mask and brain boundary. The tumor region is extracted from this brain part of the images. We found that level set evolution combining global smoothness with the flexibility of topology changes offers significant advantages over the conventional statistical classification followed by mathematical morphology. We used a modified fully automated competition based level set snake method to segment the tumor from brain cells. The tumor region which is extracted from this phase is used for $3 \mathrm{D}$ visualization. We used rendering algorithm for 3D visualization of head, brain and tumor.

Up till now we have extracted the tumor region very effectively. Surgery for brain tumor is a very critical task. First step of this is to have an idea of the brain tumor location, size, its growth rate and its thickness. We have provided a first step of the brain tumor surgery which extracts the location of the brain tumor. And gives the visualization of its structure. Future work of this research is to measure the size and growth rate of the tumor extracted region.

\section{Acknowledgements}

This research was supported by Seoul R \& BD Program (JP090972M0214832).

\section{REFERENCES}

[1] Pal, N.R. and S.K. Pal, "A review on image segmentation techniques," Pattern Recognition 26(9): 1277-1294,1993.

[2] Milan Sonka, Satish K. Tadikonda, Steve M. Collins,Knowledge-Based Interpretation of MR Brain Images, in IEEE Transaction on Medical Imaging, Vol. 15, no. 4, pp. 443-452, Aug 1996.

[3] W. Grimson, G. Ettinger, T. Kapur, M. Leventon, W. Wells, R. Kikinis, Utilizing segmented MRI data in image-guided surgery, International Journal of Pattern Recognition and Artificial Intelligence 11 (8) (1998) 1367-1397. 
[4] E. Grimson, M. Leventon, G. Ettinger, A. Chabrerie, S. Nakajima, F. Ozlen, H. Atsumi, R. Kikinis, P. Black, Clinical Experience with a High Precision Image-Guided Neurosurgery System, MICCAI, Springer, Berlin, 1998. pp. 63-73.

[5] A. Liew, and H. Yan, "Current Methods in the Automatic Tissue Segmentation of 3D Magnetic Resonance Brain Images," Current Medical Imaging Reviews, vol. 2, 2006, pp. 91-103, doi: $10.1118 / 1.1569270$.

[6] N. Duta, and M. Sonka, "Segmentation and Interpretation of MR Brain Images: An Improved Active Shape Model," IEEE Trans. Med. Imag., vol. 17, Dec. 1998 , pp. 1049-1062, doi: 10.1109/42.746716

[7] H. Li, A. Yezzi, and L. D. Cohen, "Fast 3D Brain Segmentation Using Dual-Front Active Contours with Optional User-Interaction," Proc. International Workshop on Computer Vision for Biomedical Image Applications, Lecture Notes in Computer Science, vol. 3765, 2005, pp. 335-345, doi: 10.1007/11569541_34

[8] R. Pohle, and K.D. Toennies, "Segmentation of Medical Images Using Adaptive Region Growing," Proc. SPIE, Medical Imaging, vol. 4322, 2001, pp. 1337-1346.

[9] I.N. Manousakas, P.E. Undrill, G.G. Cameron, and T.W. Redpath, "Split-and-Merge Segmentation of Magnetic Resonance Medical Images: Performance Evaluation and Extension to Three Dimensions," Computers and Biomedical Research, vol. 31, Dec. 1998, pp. 393-412, doi:10.1006/cbmr.1998.1489

[10] G. Bueno, O. Musse, F. Heitz, and J. P. Armspach, "3D Watershedbased Segmentation of Internal Structures within MR Brain Images," Proc. SPIE, Medical Imaging 2000: Image Processing, vol. 3979, 2000, pp. 284-293.

[11] K. Held, E.R. Kops, B.J. Krause, W.M. Wells, R. Kikinis, and H.W. Muller-Gartner, "Markov Random Field Segmentation of Brain MR Images,” IEEE Trans. Med. Imag., vol. 16, Dec. 1997, pp. 878-886, doi: $10.1109 / 42.650883$

[12] M. Ibrahim, N. John, M. Kabuka, and A. Younis, "Hidden Markov models-based 3D MRI brain segmentation," Image and Vision Computing, vol. 24, Oct. 2006, pp. 1065-1079, doi:10.1016/j.imavis.2006.03.001

[13] M. Desco, J. D. Gispert, S. Reig, A. Santos, J. Pascau, N. Malpica, P. Garcia-Barreno, "Statistical Segmentation of Multidimensional Brain Datasets," Proc. SPIE, Medical Imaging 2001: Image Processing, vol. 4322, Jul. 2001, pp. 184-193, doi:10.1117/12.431071

[14] R. Sammouda, N. Niki, and H. Nishitani, “A Comparison of Hopfield Neural Network and Boltzmann Machine in Segmenting MR Images of the Brain," IEEE Trans. Nucl. Sci., vol. 43, Dec. 1996, pp. 3361-3369, doi: 10.1109/NSSMIC.1995.510462

[15] J. Zhou, K. L. Chan, V. F. H. Chongand, and S. M. Krishnan, "Extraction of Brain Tumor from MR Images Using One-class Support Vector Machine," Proc. IEEE Engineering in Medicine and Biology Society (EMBS 05), 2005, pp. 6411-6414.

[16] S. Shen, W. Sandham, M. Granat, and A. Sterr, "MRI Fuzzy Segmentation of Brain Tissue Using Neighborhood Attraction with Neural-Network Optimization," IEEE Trans. Med. Imag., vol. 9, Sep. 2005, pp. 459-467, doi: 10.1109/TITB.2005.847500

[17] W. R. Crum, "Spectral Clustering and Label Fusion for 3D Tissue Classification: Sensitivity and Consistency Analysis," Proc. Medical Image Understanding and Analysis, July, 2008, pp. 149-153.

[18] M. Arfan Jaffar, Bilal Ahmed, Nawazish Naveed, Ayyaz Hussain, and Anwar M. Mirza, "Fuzzy c-means clustering with spatial information for Color image segmentation", Third International Conference on Electrical Engineering (ICEE), 2009.

[19] Xiangrong Zhang, Feng Dong, Gordon Clapworthy, Youbing Zhao, Licheng Jiao, "Semi-supervised Tissue Segmentation of 3D Brain MR Images", 14th International Conference Information Visualisation, ISSN: 1550-6037, 26-29 July 2010, pages: 623 - 628

[20] S.M. Smith, "Fast robust automated brain extraction," Human Brain Mapping, vol. 17, no. 3, pp. 143-155, Nov 2002.

[21] A.J. Bartsch, G. Homola, A. Biller, S.M. Smith, H.G. Weijers, and et al Wiesbeck, G.A., "Manifestations of early brain recovery associated with abstinence from alcoholism.," Brain, vol. 130, pp. 3647, 2007.
[22] M. Battaglini, S.M. Smith, S. Brogi, and N. De Stefano, "Enhanced brain extraction improves the accuracy of brain atrophy estimation," NeuroImage, vol. 40, no. 2, pp. 583-589, April 2008.

[23] Ivana Despotovic, Ewout Vansteenkiste, Wilfried Philips, "Brain volume segmentation in newborn infants using multi-modal MRI with a low inter-slice resolution", International Conference of the IEEE EMBS Buenos Aires, ISSN: 1557-170X, September, 2010.

[24] Konstantin Levinski, Alexei Sourin and Vitali Zagorodnov, "3D VISUALIZATION AND SEGMENTATION OF BRAIN MRI DATA", International Conference on Computer Graphics Theory and Applications (GRAPP), 2009.

[25] Kang, Y., K. Engelke, Willi A. Kalender,"Interactive 3D editing tools for image segmentation", Medical Image Analysis, Volume 8, Issue 1, March 2004, Pages 35-46.

[26] El-Sayed A, El-Dahshan, Abdel-Badeeh M.Salem and Tamer H.Younis, "A hybrid technique for automatic MRI brain images classification", Studia Univ, Babes Bolyai,Informatica, Vol LIV,2009

[27] Sriparna Saha and Sanghamitra Bandyopadhyay, "MRI Brain Image Segmentation by Fuzzy Symmetry Based Genetic Clustering Technique", Evolutionary Computation, 2007, pp .4417-4424

[28] Yongyue Zhang, Michael Brady, and Stephen Smith, 'Segmentation of Brain MR Images Through a Hidden Markov Random Field Model and the Expectation-Maximization Algorithm", IEEE Transaction on Medical Imaging, vol.20, No. 1,2001, pp. 45-57

[29] R.Ratan,S.Sharma, S.k.Sharamac, "Brain tumor detection based on multi-parameter MRI image analysis", ICGST-GVIP Journal, ISSN 1687-398X, Vol. 9, Issue III, 2009, pp. 9-17

[30] R. B. Dubey, M. Hanmandlu and S. K. Gupta, "Semi-automatic Segmentation of MRI Brain Tumor" , ICGST-GVIP Journal, Vol 9, Issue 4, ISSN: 1687-398X, 2009, pp.33-40

[31] Michela Antonelli, Beatrice Lazzerini, Francesco Marcelloni. "Segmentation and reconstruction of the lung volume in CT images", ACM Symposium on Applied Computing, 2005.

[32] Pietro Perona and Jitendra Malik, "Scale-space and edge detection using anisotropic diffusion", IEEE Trans. on Pattern Analysis and Machine Intelligence, 12(7):629-639, July 1990.

[33] Tom M. Mitchell, Machine Learning , Bayesian Learning ,McGraw Hill, 1997,pp.154-178

[34] Micheal Kass, Andrew Witkin, and EemetriTerzopoulos. Snakes: Active Contour Models. International Journal of Computer Vision, pages 321 $331,1988$.

[35] F. Maes, A. Collignon, D. Vandermeulen, G. Marchal and P. Suetens, "Multi-modality image registration by maximization of mutua information," IEEE Transactions on Medical Imaging, vol. 16, no. 2, pp. $187-198,1997$.

[36] E. Praun, H. Hoppe, A. Finkelstein.Robust mesh watermarking.ACM SIGGRAPH 1999 Conference Proceedings, 69-76.

[37] H. Hoppe, T. DeRose, T. Duchamp, J. McDonald, W. Stuetzle, Mesh optimization. ACM SIGGRAPH 1993 Conference Proceedings, 19-26.

[38] Francis S. Hill, Stephen M. Kelley, "Computer graphics using OpenGL", Volume 2006

[39] Herman, G.T.; Zheng, J.; Bucholtz, C.A., "Shape-based Interpolation," IEEE Computer Graphics and Applications, ISSN: 02721716, May 1992, pages: 69 - 79.

[40] Robert A. Drebin, Loren Carpenter, Pat Hanrahan, "Volume Rendering," SIGGRAPH '88 Proceedings of the 15th annual conference on Computer graphics and interactive techniques ISBN:0-89791-275-6, 1988.

[41] Harvard Medical School, Web: data available at http://med.harvard.edu/ AANLIB/.

[42] Abrar MRI \&CT Center, Rawalpindi, Pakistan: Data available at http://www.abrarmrict.com/ 


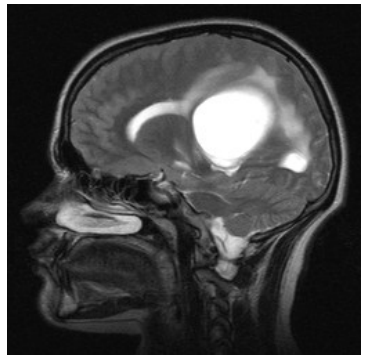

(a) Original Image

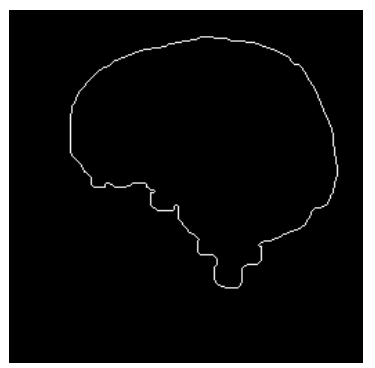

(e) Brain Mask Boundary

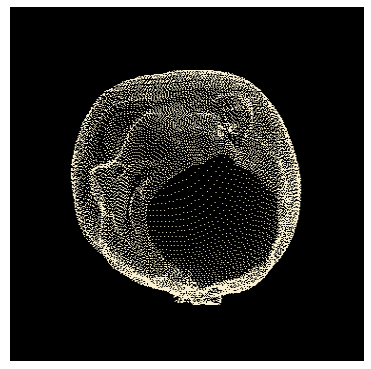

(i) Head Boundary

Points Placement

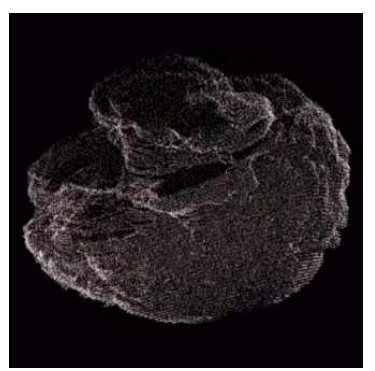

(m) Brain 3D Model

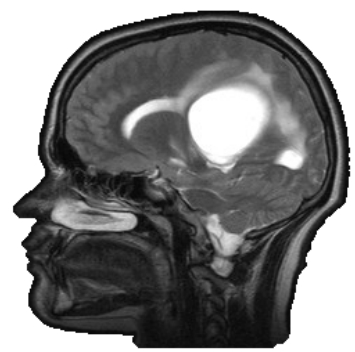

(b) Head Mask

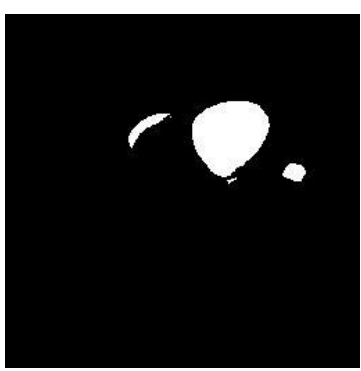

(f) Tumor Mask

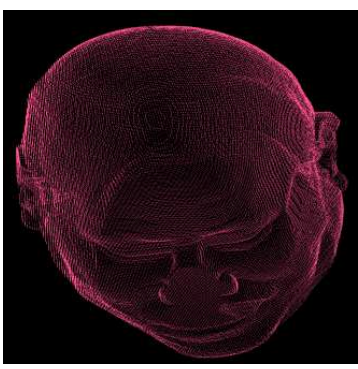

(j)Head Boundary

Points Placement

(Other View)

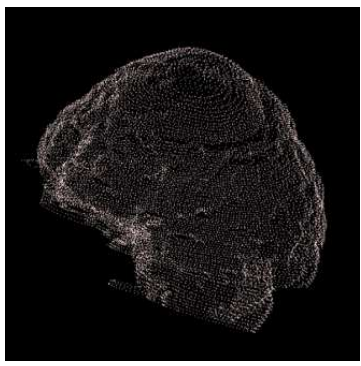

(n) Brain 3D Model (Another View)

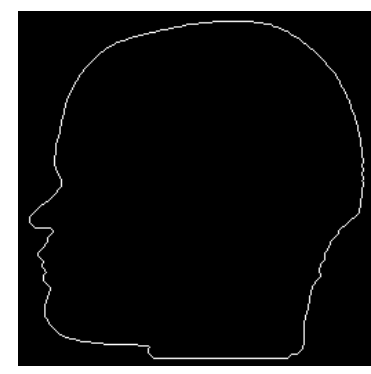

(c) Head Mask Boundary

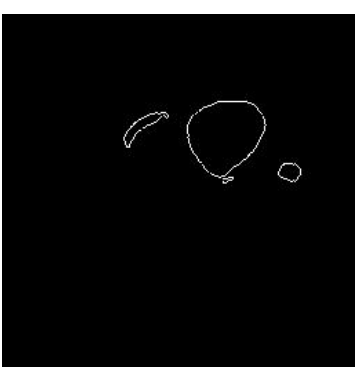

(g) Tumor Mask Boundary

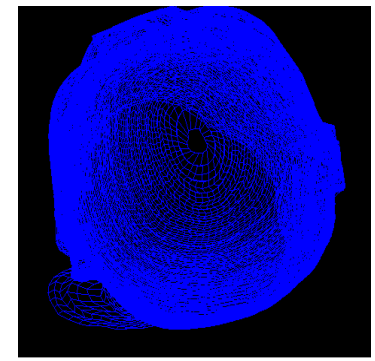

(k) Head Mesh Model after interpolation

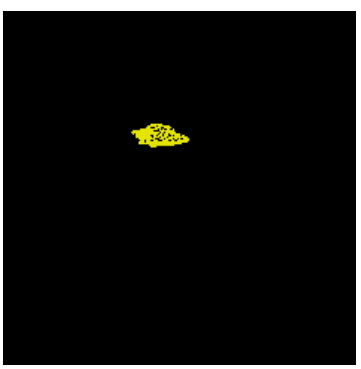

(o) Tumor 3D Model

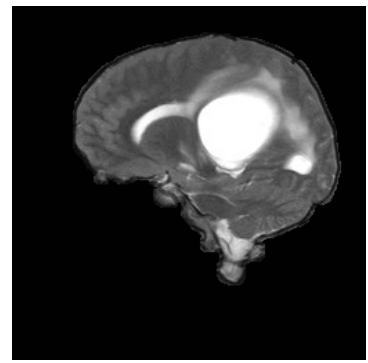

(d) Brain Mask

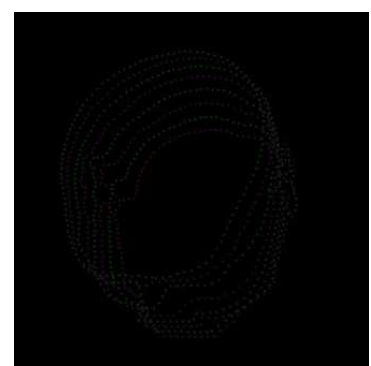

(h) Pattern of Boundary pixels

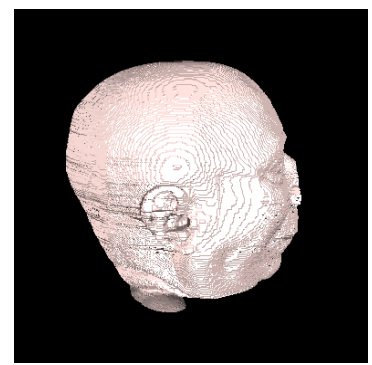

(l) Rendered 3D Head Model

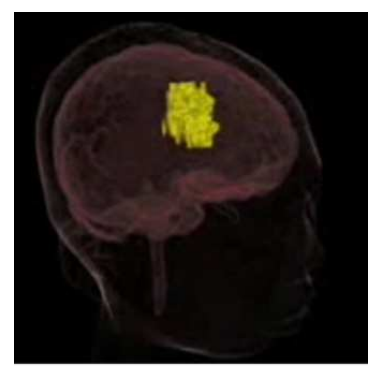

(p) Final Integrated 3D Model

Fig. 12 Extraction of Head, Brain and Tumor Boundaries and their 3D Modeling 